Revista de

CIENCIAS AMBIENTALES

Tropical Journal of Environmental Sciences

\title{
Estudio preliminar para la determinación de plaguicidas en vegetales comercializados en una zona de Michoacán (México)
}

Preliminary study for the determination of pesticides in vegetables marketed in a zone of Michoacán (México)

\section{Rocío Ramírez-Jiménez ${ }^{a}$, Ernesto Oregel-Zamudio ${ }^{b}$}

a Especialista en Ciencias Ambientales, docente e investigadora del Instituto Politécnico Nacional, Centro Interdisciplinario de Investigación para el Desarrollo Integral Regional (CIIDIR), Unidad Michoacán, México, mdramirez@ipn.mx

b Especialista en Producción Agrícola Sustentable, docente e investigador del Instituto Politécnico Nacional, Centro Interdisciplinario de Investigación para el Desarrollo Integral Regional (CIIDIR), Unidad Michoacán, México, eoregel@ipn.mx, ORCID: https://orcid. org/0000-0002-5015-5137

Director y Editor:

Dr. Sergio A. Molina-Murillo

Consejo Editorial:

Dra. Mónica Araya, Costa Rica Limpia, Costa Rica Dr. Gerardo Ávalos-Rodríguez. SFS y UCR, USA y Costa Rica Dr. Olman Murillo Gamboa, ITCR, Costa Rica

Dr. Luko Hilje, CATIE, Costa Rica

Dr. Arturo Sánchez Azofeifa. Universidad de Alberta-Canadá

Asistente:

Joseline Jimenez Brenes

Editorial:

Editorial de la Universidad Nacional de Costa Rica (EUNA)
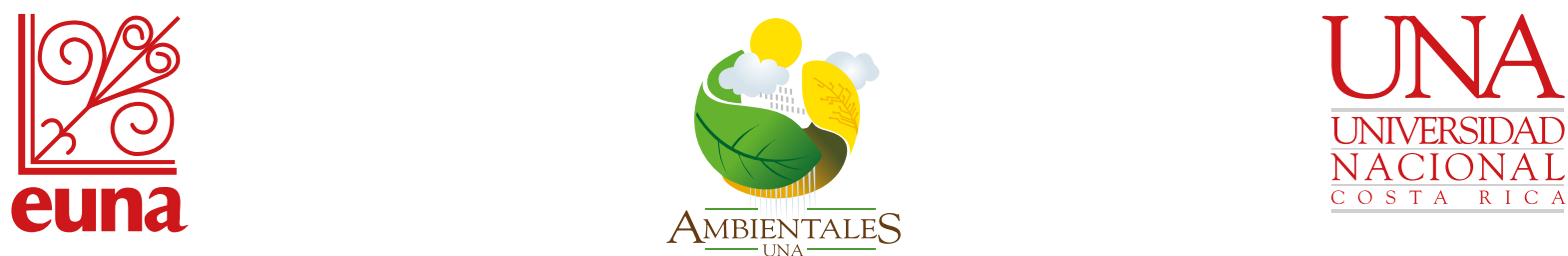

Los artículos publicados se distribuyen bajo una Creative Commons Reconocimiento al autor-No comercial-Compartir igual 4.0 Internacional (CC BY NC SA 4.0 Internacional) basada en una obra en http://www.revistas.una.ac.cr/ambientales, lo que implica la posibilidad de que los lectores puedan de forma gratuita descargar, almacenar, copiar y distribuir la versión final aprobada y publicada (post print) del artículo, siempre y cuando se realice sin fines comerciales, no se generen obras derivadas y se mencione la fuente y autoría de la obra. 


\title{
Estudio preliminar para la determinación de plaguicidas en vegetales comercializados en una zona de Michoacán (México)
}

\author{
Preliminary study for the determination of pesticides in vegetables marketed in a \\ zone of Michoacán (México)
}

Rocío Ramírez-Jiménez $z^{a}$ Ernesto Oregel-Zamudio ${ }^{b}$

[Recibido: 26 de febrero, 2018. Aceptado: 22 de marzo, 2018. Corregido: 16 de abril, 2018. Publicado: 1 de julio, 2018.]

Resumen

Los organofosforados (OF) son ampliamente utilizados en el mundo por su bajo costo, alta eficacia y menor persistencia. Estos compuestos neurotóxicos han sido detectados en matrices biológicas y ambientales en diversos países. El uso de plaguicidas implica el riesgo de acumulación de residuos en productos agrícolas; no obstante, en algunas regiones de México, el monitoreo de residuos de plaguicidas en alimentos y en otras matrices es limitado. El objetivo de este trabajo fue determinar residuos de OF en vegetales comercializados en tianguis de Jiquilpan, Michoacán. Se recolectaron muestras de calabaza, pepino, jitomate, cebolla, fresa y manzana $(n=30)$, además de jitomate y fresa orgánicos $(n=6)$. Se realizó la extracción de las muestras con acetato de etilo y se analizó la presencia de residuos de malatión y clorpirifos-etílico por cromatografía en capa fina de alta resolución (HPTLC). Las muestras orgánicas no presentaron residuos de malatión y clorpirifos-etílico. Bajo las condiciones de este estudio, no se detectaron residuos de malatión en las muestras de vegetales; sin embargo, se observó la presencia de residuos de clorpirifos-etílico en 4 muestras de cebolla y en 1 de manzana ( $20 \%$ del total de muestras). La técnica de HPTLC se utilizó como una técnica de cribado. Se ha reportado que la exposición crónica a bajas concentraciones de plaguicidas puede representar un riesgo a la salud humana, ya que sus efectos pueden ser aditivos y pueden manifestarse largo plazo. Se requiere el monitoreo periódico de plaguicidas en alimentos y poblaciones expuestas.

Palabras clave: cultivos agrícolas; HPTLC; inocuidad alimentaria; organofosforados.

\section{Abstract}

Organophosphates (OP) are widely used in the world for their low cost, high efficiency, and less persistence. These neurotoxic compounds have been detected in biological and environmental matrices in various countries. The use of pesticides implies the risk of accumulation of residues in agricultural products; however, in some regions of Mexico, the monitoring of pesticide residues in food and other matrices is limited. The objective of this work was to determine residues of OP in vegetables commercialized in tianguis (popular market days) of Jiquilpan, Michoacan, Mexico. Samples of pumpkin, cucumber, tomato, onion, strawberry, and apple were collected $(n=30)$, as well as tomato and organic strawberry $(n=6)$. The samples with ethyl acetate were extracted, and the presence of malathion and chlorpyrifos-ethyl residues was analyzed using high-resolution thin layer chromatography (HPTLC). The organic samples did not show residual malathion and chlorpyrifos-ethyl. Under the conditions of this study, no malathion residues were detected in the vegetable samples. However, the presence of chlorpyrifos-ethyl residues was observed in four onion samples and in one apple sample ( $20 \%$ of the total samples). The HPTLC technique was

a Especialista en Ciencias Ambientales, docente e investigadora del Instituto Politécnico Nacional, Centro Interdisciplinario de Investigación para el Desarrollo Integral Regional (CIIDIR), Unidad Michoacán, México, mdramirez@ipn.mx

b Especialista en Producción Agrícola Sustentable, docente e investigador del Instituto Politécnico Nacional, Centro Interdisciplinario de Investigación para el Desarrollo Integral Regional (CIIDIR), Unidad Michoacán, México, eoregel@ipn.mx, ORCID: https://orcid. org/0000-0002-5015-5137

( 
used as a screening technique. It has been reported that chronic exposure to low concentrations of pesticides can represent a risk to human health, since its effects could be additive and could appear in the medium and long term. Periodic monitoring of pesticides in food and exposed populations is required.

Keywords: Agricultural crops, HPTLC, food safety, organophosphates.

\section{Introducción}

Según la Comisión Federal para la Protección contra Riesgos Sanitarios (COFEPRIS, 2016), un plaguicida es cualquier sustancia o mezcla de sustancias destinadas a prevenir, destruir, repeler, o mitigar cualquier plaga, $y$, principalmente, se utilizan para controlar las plagas en los campos agrícolas. De acuerdo con su estructura química, los plaguicidas se clasifican principalmente en carbamatos, piretroides, triazinas, neonicotinoides, organoclorados, organofosforados, entre otros. Los organofosforados (OF) son un grupo de insecticidas ampliamente utilizado en el mundo, debido a su bajo costo, alta eficacia y menor persistencia (Kazemi et al., 2012). Estos se degradan rápidamente al exponerse a la luz solar, al aire y al suelo, y algunos de ellos tienen una alta volatilidad que limita su persistencia después de la aplicación foliar; sin embargo, ciertos OF pueden permanecer en el suelo como residuos, cuando son asperjados sobre cultivos, además de que pueden llegar a los cuerpos de aguas superficiales y subterráneas por escorrentías o lixiviación del suelo. Algunos de los OF más utilizados son clorpirifos, malatión, diazinón, metamidofos, paratión-metílico, dimeotato, monocrotofos, etión, coumafos, fenitrotion, cianofos, entre otros (Androutsopoulos et al., 2013).

El mecanismo de toxicidad de los OF consiste en la inhibición de las colinesterasas, enzimas cuya función biológica es la hidrólisis de la acetilcolina, neurotransmisor implicado en la transmisión del impulso nervioso en las membranas sinápticas (Androutsopoulos et al., 2013). Los efectos tóxicos de los OF por intoxicaciones agudas han sido documentados por varios investigadores; mientras que aquellos por la exposición crónica son menos conocidos. Se han reportado daños en el desarrollo, inmunológicos, reproductivos, endocrinos y genotóxicos, alteraciones neuroconductuales y cáncer (Androutsopoulos et al., 2013). Respecto a los efectos neurotóxicos de los OF, si la exposición ocurre durante alguna etapa sensible del desarrollo, aun en bajos niveles de exposición, se ha indicado que pueden interferir con el desenvolvimiento del cerebro, generar riesgo de daños permanentes que podrían surgir en etapas posteriores como la adolescencia y edad adulta (Bouchard et al., 2011).

Los métodos convencionales para la determinación de residuos de plaguicidas en alimentos son la cromatografía de gases (CG) y la cromatografía de líquidos de alta resolución (HPLC) acopladas a espectrometría de masas y a otros detectores como el de nitrógeno-fósforo, captura de electrones y fotométrico de llama (Fenik et al., 2011). La cromatografía en capa fina de alta resolución (HPTLC, por sus siglas en inglés) es una técnica de detección simple, barata, cualitativa y cuantitativa que permite el análisis simultáneo de varias muestras en una sola placa, así como la posibilidad de repetir la detección y cuantificación por otras técnicas, utilizando la fracción que se almacena en la placa (Sherma, 2017). La HPTLC combinada con detección quimioselectiva se emplea en la determinación de OF, usando reactivos como yodo, molibdato de amonio, nitrato de

Creative Commons (Reconocimiento al autor-No comercial-Compartir igual 4.0 Internacional
(CC BY NC SA 4.0 Internacional)


plata, cloruro de paladio y 2.6-dibromo-benzoquinona-4-clorimina (DCQ) (Obare et al., 2010). También se utilizan inmunoensayos, aplicando enzimas como las colinesterasas y la glucosa oxidasa (Androutsopoulos et al., 2013). En los últimos años, la HPTLC tiene mejoras importantes como su acoplamiento a CG y HPLC, lo que permite la detección cuantitativa más eficiente de residuos de plaguicidas en frutas y hortalizas, a través de métodos multi-residuos (Sherma, 2017). El análisis de plaguicidas sigue siendo una de las principales aplicaciones de la HPTLC para el análisis de alimentos, así como de muestras ambientales y biológicas (Sherma, 2017).

El ser humano puede estar expuesto directamente a los OF y a otros plaguicidas asperjados en el ambiente a través de la deriva y deposición de aerosoles y vapores, al entrar en contacto con suelo, polvo y materiales contaminados, por el uso residencial y urbano de insecticidas e, indirectamente, a través de la ingestión de alimentos y agua contaminados con residuos de plaguicidas (Damalas y Eleftherohorinos, 2011). Bajo este escenario, la población en general está potencialmente expuesta a plaguicidas; sin embargo, aquellos que viven en áreas agrícolas se encuentran en mayor riesgo.

La principal vía de exposición a los OF es la ingestión de alimentos contaminados (Damalas y Eleftherohorinos, 2011). Los alimentos pueden contaminarse con plaguicidas durante la producción, el transporte o el almacenamiento. Diversos estudios han demostrado que la determinación de residuos de plaguicidas en alimentos es una buena forma de evaluar la exposición humana a plaguicidas, considerando que algunos alimentos pueden tener un mayor impacto en los niveles de exposición (Chen et al., 2012).

La Unión Europea (UE), Estados Unidos de América, Canadá y otros países cuentan con programas de monitoreo que evalúan periódicamente los residuos de plaguicidas en alimentos, con base en límites máximos de residuos (LMR). En este sentido, la Autoridad Europea de Seguridad Alimentaria (EFSA, 2017), en el 2015, analizó 774 plaguicidas en muestras de vegetales de países europeos (69.3 \%) y de productos importados $(25.8 \%)$. En el $53.3 \%$ de las muestras, no se encontró residuos de plaguicidas, mientras que el $43.9 \%$ presentó residuos por debajo de los LMR, y solo el $2.8 \%$ excedió los LMR.

En el 2011, Dinamarca, Finlandia, Noruega y Suecia determinaron residuos de plaguicidas en 63 tipos de frutas y verduras importadas de Bangladesh, Indonesia, Corea, Laos, Malasia, Nepal, Filipinas, Paquistán, Tailandia y Vietnam (Skretteberg et al., 2015). En el 60 \% de las muestras, no se halló residuos de plaguicidas, mientras que en el $28 \%$ se encontró residuos en concentraciones menores o iguales a los LMR y, en el $12 \%$, mayores a los LMR. El clorpirifos se encontró en el $7.4 \%$ de las muestras, de las cuales el 32.1\% tuvo niveles por arriba de los LMR, principalmente, en pitaya/guayaba y albahaca.

En Estados Unidos de América, la Administración de Alimentos y Drogas (FDA, por sus siglas en inglés) realiza, anualmente, un Programa de Monitoreo de Residuos de Plaguicidas (PMRP) en alimentos tanto de origen nacional como de importación. En el PMRP del 2015 (FDA, 2015), no se detectaron residuos de plaguicidas en las muestras de frutas y vegetales analizadas ( 18.3 y $38 \%$, respectivamente), y solo el 2.2 y $3.8 \%$ presentó residuos por encima de los LMR. 207 plaguicidas fueron identificados en las muestras y los OF más frecuentemente detectados fueron el clorpirifos-etílico (7.4\%), el clorpirifos-metílico (10.3\%) y el malatión (16.1\%). 
En el 2007, países nórdicos realizaron el análisis de plaguicidas en frutas y vegetales importados de Argentina, Brasil, Chile, Colombia, Ecuador, Perú, Suriname y Uruguay (Hjorth et al., 2011). El 19 \% de las muestras no presentó residuos de plaguicidas, el 72 \% mostró residuos por debajo de los LMR y el 8.4 \% tuvo residuos mayores a los LMR. Los países con mayor porcentaje de muestras con residuos arriba de los LMR fueron Brasil, Uruguay y Colombia. Se detectó un total de 83 plaguicidas; de estos tiabendazol, imazalil y clorpirifos fueron los determinados con mayor frecuencia $(29.3,25.4$ y $16.9 \%$, respectivamente). Los mayores porcentajes de muestras por arriba de los LMR fueron de manzana, papaya, maracuyá y uvas. El plaguicida encontrado en mayor concentración fue la iprodiona $(6.6 \mathrm{mg} / \mathrm{kg})$ en una muestra de melocotón de Chile; y la más alta concentración por arriba de un LMR se encontró en una muestra de maracuyá de Colombia, que contenía ditiocarbamatos. En general, las manzanas de los países nórdicos presentaron menos residuos de plaguicidas que las de Sudamérica.

En México, se emplean varios OF como el clorpirifos, malatión, paratión-metílico, metamidofós y diazinón; no obstante, no hay registros claros sobre las cantidades que se utilizan (Hernández-Antonio y Hansen, 2011). Por otro lado, aún se emplean algunos OF que son prohibidos en otros países, como el azinfos-metílico, forato, fosfamidón, metidatión, metamidofos, mevinfos, monocrotofos, omeotato y paratión-metílico. El Centro Nacional de Residuos y Contaminantes (CNRPYC) reportó la presencia de residuos de 19, 15 y 17 plaguicidas no autorizados en los monitoreos del 2005, 2006 y 2007, respectivamente (Pérez et al., 2013). Algunos de los plaguicidas más frecuentemente encontrados fueron $\mathrm{OF}$ como el ometoato, methamidofos, dimetoato y clorpirifos. Por otro lado, Suárez-Jacobo et al. (2017) encontraron residuos de 15 plaguicidas en naranjas de huertos de Nuevo León, en niveles por debajo de los LMR de EUA y Japón; sin embargo, el $11 \%$ de las muestras presentó concentraciones por arriba de los LMR de la UE. Entre los OF detectados con mayor frecuencia estuvieron el malatión (15\%) y el clorpirifos-etílico (16\%), los cuales, además, tuvieron las concentraciones más altas.

De acuerdo con informes de monitoreo de otros países, el clorpirifos-etílico y el malatión son dos de los OF que se han encontrado en frutas y vegetales. Estos OF son utilizados en México y en la región de estudio (Pérez-Olvera et al., 2011); sin embargo, las investigaciones sobre los niveles de plaguicidas tanto en alimentos como en otras matrices biológicas y ambientales aún son escasas, por lo que es necesaria la realización de estudios de monitoreo de residuos de plaguicidas en estas matrices, particularmente, en productos agrícolas de consumo nacional y local. El objetivo de este trabajo fue determinar residuos de clorpirifos-etílico y malatión en vegetales comercializados en tianguis de Jiquilpan, Michoacán.

\section{Materiales y métodos}

\subsection{Descripción y localización de la zona de estudio}

El municipio de Jiquilpan tiene una superficie de $242.13 \mathrm{~km}^{2}$ y se localiza al noroeste del Estado de Michoacán, México. Pertenece a la región Ciénega de Michoacán en las coordenadas 1959’ de latitud norte y $102^{\circ} 43^{\prime}$ de longitud oeste, a una altura de $1550 \mathrm{~m}$ sobre el nivel del mar. Su clima es

Creative Commons (Reconocimiento al autor-No comercial-Compartir igual 4.0 Internacional
(CC BY NC SA 4.0 Internacional)


templado con lluvias en verano (de mayo a octubre), con temperaturas que oscilan de 10.4 a $25.4^{\circ} \mathrm{C}$ y una temperatura media anual de $19.4^{\circ} \mathrm{C}$. Tiene una precipitación pluvial anual de $826 \mathrm{~mm}$. La época seca se presenta entre noviembre y abril; corresponde el mes más seco a marzo. La población total en Jiquilpan es de 32950 (INEGI, 2015). Las actividades económicas predominantes en la región son la agricultura y la ganadería. Los principales cultivos son: maíz, sorgo, garbanzo, trigo, cártamo, cebolla, jitomate (Solanum licopersicum), tomate verde (Physalis ixocarpa) chile, entre otros.

\subsection{Equipos y materiales}

Se utilizó un equipo de cromatografía de capa fina de alta resolución (HPTLC, CAMAG, Muttenz, Suiza), equipado con un automuestreador (ATS4, CAMAG, Muttenz, Suiza), una cámara de desarrollo automática (ADC2, CAMAG, Muttenz, Suiza), un visualizador (CAMAG, Muttenz, Suiza) y un software para el procesamiento de placas de HPTLC (visión CATS 2.1, CAMAG, Muttenz, Suiza). Además, se empleó un dispositivo de inmersión para placas de cromatografía de capa fina (CAMAG, Muttenz, Suiza) y un calentador de placas (CAMAG, Muttenz, Suiza). Para la concentración de las muestras, se usó un evaporador (TurboVap, LV, Biotage, Suecia). Para el procesamiento de las muestras, se utilizó un procesador de alimentos (465-4, Osterizer, México) y una campana de extracción. También se trabajó con un vortex (Maxi-Mix, Barnstead/Thermolyne, Dubuque, Iowa, USA), una balanza analítica digital Sartorius. Para la cromatografía en capa fina de alta resolución, fueron colocadas placas de HPTLC de silica gel 60 F254 $(20$ x 10 cm), Merck.

\subsection{Reactivos y disoluciones}

Se utilizaron como estándares clorpirifos [O,O-dietil 0-(3, 5, 6-tricloro-2-piridil) fosforotioato], marca Pestanal analytical standard y Sigma-Aldrich 45395-250 mg, 99.9 \%, y malatión [O,O-dimetil fosforoamidotioato de dietil mercapto succinato], grado técnico, $83.7 \%$ (1 $000 \mathrm{~g}$ de i.a./L). Como solventes, se emplearon acetato de etilo, metanol, acetona y hexano, todos grado HPLC (Mallinckrodt Laboratory Chemicals, Mallinckrodt Baker, USA). Se utilizó sulfato de sodio anhidro $\left(\mathrm{Na}_{2} \mathrm{SO}_{4}\right.$ ), marca Sigma-Aldrich, sometido a $450{ }^{\circ} \mathrm{C}$, durante 8 horas, almacenado en una botella herméticamente cerrada en un desecador hasta su uso; carbón activado, Merck, activado a $105{ }^{\circ} \mathrm{C}$ durante 4 horas (Iqbal et al., 2009; Munawar y Hameed 2013); cloruro de sodio $(\mathrm{NaCl})$, marca JT Baker; cloruro de paladio $\left(\mathrm{PbCl}_{2}\right)$ al $0.5 \%$ (en $100 \mathrm{~mL}$ de $\mathrm{HCl}$ al $10 \%$ ); disoluciones stock de clorpirifos y malatión (10000 mg/L), preparadas en metanol y almacenadas en viales ámbar de vidrio a $-20^{\circ} \mathrm{C}$; disoluciones estándar de clorpirifos y malatión (100 y 1 $000 \mathrm{mg} / \mathrm{L}$ ), preparadas en metanol a partir de diferentes volúmenes de las disoluciones stock de $10000 \mathrm{mg} / \mathrm{L}$ y almacenadas en viales ámbar de vidrio a $4^{\circ} \mathrm{C}$ hasta el momento de su uso.

\subsection{Colecta de muestras}

En noviembre de 2016, se colectaron muestras de jitomate saladette, cebolla, calabacita, pepino, fresa y manzana, en los tres tianguis y en el mercado local de la zona de estudio. Adicionalmente, se adquirieron vegetales orgánicos: jitomate saladette en una tienda comercial y fresas orgánicas (certificadas) con un productor de la región. La colecta de las muestras se realizó de 
acuerdo con el procedimiento de la Directiva 2002/63/CE de la Unión Europea. En cada sitio, se adquirieron $2 \mathrm{~kg}$ de cada vegetal con 3 locatarios elegidos al azar. Las muestras se guardaron en bolsas cerradas herméticamente. Se mantuvieron en refrigeración a $4{ }^{\circ} \mathrm{C}$ hasta el momento de su análisis. Las muestras de vegetales se lavaron con agua y jabón como normalmente un consumidor lo hace. Finalmente, se cortaron en trozos de 2-3 mm, con ayuda de un procesador de alimentos. Se integraron muestras compuestas por tipo de fruta o vegetal.

\subsection{Preparación y extracción de muestras}

Se tomaron porciones representativas de cada una de las muestras vegetales y se sometieron a extracción sólido-líquido, respectivamente, de acuerdo con Munawar y Hameed (2013). Se colocaron 60 $\mathrm{g}$ de muestra en un matraz Erlenmeyer de $250 \mathrm{~mL}$. A continuación, fueron agregados $20 \mathrm{~g} \mathrm{de} \mathrm{Na}_{2} \mathrm{SO}_{4}$ anhidro, $2.5 \mathrm{~g}$ de $\mathrm{NaCl}$ y $70 \mathrm{~mL}$ de acetato de etilo; después, se sometieron a vibración mecánica durante $60 \mathrm{~min}$ a temperatura ambiente $\left(25^{\circ} \mathrm{C}\right)$. Se permitió que las fases se separaran durante $30 \mathrm{~min}$ y se recuperó la orgánica. Posteriormente, los extractos se filtraron a través de un embudo de vidrio con algodón y fibra de vidrio, para eliminar restos de material vegetal; enseguida, se filtraron en un embudo de vidrio sobre papel filtro Whatman 42 y carbón activado. Los extractos obtenidos se transfirieron a tubos cónicos y se evaporaron a sequedad a $30-35^{\circ} \mathrm{C}$ bajo corriente suave de nitrógeno. Las muestras secas se resuspendieron en acetona $(2 \mathrm{~mL})$ y se guardaron en viales de $2-4 \mathrm{~mL} \mathrm{a}-20^{\circ} \mathrm{C}$.

\subsection{Cromatografía en capa fina de alta resolución (HPTLC)}

La cromatografía se realizó de acuerdo con Jaiswal et al. (2008b) sobre placas de HPTLC de silica gel previamente lavadas en metanol, secadas y activadas a $105^{\circ} \mathrm{C}$ durante $30 \mathrm{~min}$. Tanto las muestras como los estándares fueron aplicados en forma de bandas de $5 \mathrm{~mm}$, usando un auto-muestreador ATS4, equipado con una jeringa de $10 \mu \mathrm{L}$; el volumen de aplicación en las pistas fue de $3 \mu \mathrm{L}$. Las distancias desde el borde inferior, desde el lado izquierdo y entre las pistas fueron de $10 \mathrm{~mm}$. Las placas se desarrollaron en una cámara de desarrollo automática $\mathrm{ADC} 2$, en equilibrio vapor/disolvente, empleando una mezcla de hexano:acetona (8:2) como fase móvil. La distancia de desarrollo fue de $70 \mathrm{~mm}$ desde el borde inferior. La actividad de la placa se ajustó a una humedad relativa del $33 \%$, mediante el uso de una disolución acuosa saturada de cloruro de magnesio $\left(\mathrm{MgCl}_{2} \cdot 6 \mathrm{H}_{2} \mathrm{O}\right)$. La placa desarrollada y seca se sumergió en la disolución de $\mathrm{PbCl}_{2}$ al $0.5 \%$, en un dispositivo de inmersión, durante $3 \mathrm{~s}$ a una velocidad de inmersión de $1 \mathrm{~cm} / \mathrm{s}$ para ser revelada. La placa se secó durante aproximadamente un minuto al aire y después se secó en un calentador de placas TLC durante 20 min a $105^{\circ} \mathrm{C}$. Las imágenes de las placas se documentaron mediante el uso del sistema de documentación DigiStore 2, con iluminación en el rango visible, en el modo de reflectancia, bajo luz blanca. Los datos obtenidos se analizaron con el Software winCATS, versión 2.1.

\section{Resultados y discusión}

En México, se denomina tianguis (del náhuatl tianguiztle) a los mercados que se establecen en la vía pública, periódicamente una vez por semana, en los que se venden productos de consumo como frutas, verduras, cereales, plantas medicinales, carne, lácteos, abarrotes, ropa y otros productos;

$44 \begin{aligned} & \text { Creative Commons (Reconocimiento al autor-No comercial-Compartir igual 4.0 Internacional } \\ & \text { (CC BY NC SA 4.0 Internacional) }\end{aligned}$


mientras que los mercados locales son espacios techados divididos en locales, en los que se expenden diversos productos perecederos y semiperecederos (Duhau y Giglia, 2007). Cabe resaltar que los vegetales que se comercializan en tianguis normalmente son cultivados a cielo abierto o en invernaderos de la región, además de que son directamente consumidos por sus pobladores. Los avances recientes en el campo de la agricultura han conducido a un renovado interés para recopilar información sobre el uso de plaguicidas en cultivos agrícolas en la región Ciénaga de Chapala.

En este estudio, se analizó la presencia de residuos de malatión y clorpirifos-etílico en muestras de frutas y vegetales provenientes del mercado local "Zaragoza" ubicado en el centro de Jiquilpan, Michoacán, así como de los tres tianguis de esta localidad: el primero se instala los días viernes a un costado del Jardín de la Paz, el segundo se ubica los sábados en las inmediaciones del estadio local y el último se sitúa los domingos en el centro de la localidad. Tanto en los tianguis como en el mercado se comercializan principalmente frutas y vegetales de procedencia local y de temporada, aunque también se pueden adquirir frutas y vegetales nacionales y algunos de importación. En el caso de las muestras de frutas y vegetales recolectadas en este estudio no se sabe con certeza si todas son de la región.

La región Ciénega de Chapala es un área compartida por los estados de Jalisco y Michoacán, ubicada al oriente del lago de Chapala y constituida por los municipios de Jamay, Ocotlán, Poncitlán, Chapala, Jocotepec, Tuxcueca y Tizapán en Jalisco; Cojumatlán de Régules, Venustiano Carranza, Briseñas, Jiquilpan, Sahuayo y Villamar en Michoacán (Sandoval-Moreno y Ochoa-Ocaña, 2010). Estudios señalan la importancia de la agricultura, como la principal actividad en esta región, y del uso intensivo de químicos para el control de plagas en cultivos. No obstante, la información sobre la aplicación de plaguicidas en los cultivos de la región es limitada, se sabe que el clorpirifos y el malatión, además de otros OF indicados en el Cuadro 1, son empleados en la zona de estudio.

Cuadro 1. Principales insecticidas organofosforados usados por los agricultores en la región Ciénega de Chapala

\begin{tabular}{|c|c|c|c|}
\hline${ }^{\mathrm{a}} \mathrm{OF}$ & a Nombre comercial & $\begin{array}{c}{ }^{\text {a }} \text { Cantidad aplicada }(\mathrm{g} / \\
\text { ha) }\end{array}$ & ${ }^{\mathrm{b}}$ Clasificación peligrosidad / $\mathrm{DL}_{50}$ \\
\hline Clorpirifos & Lorsban, Foley rey $33.8 \%$ & 140.5 & $\begin{array}{l}\text { II / } 135 \mathrm{mg} / \mathrm{kg} \\
\text { (moderadamente peligroso) }\end{array}$ \\
\hline Paratión metílico & Flash $2 \%$, Folodol-M72, Penncap-M & 127.3 & $\begin{array}{l}\text { IA / } 14 \mathrm{mg} / \mathrm{kg} \\
\text { (extremadamente peligroso) }\end{array}$ \\
\hline Metamidofos & Tamaron 43.3, Tramofos & & $\begin{array}{l}\text { IB / } 30 \mathrm{mg} / \mathrm{kg} \\
\text { (altamente peligroso) }\end{array}$ \\
\hline Dimetoato & Dimetoato, Cygon & 9.6 & $\begin{array}{l}\text { II / } 150 \mathrm{mg} / \mathrm{kg} \\
\text { (moderadamente peligroso) }\end{array}$ \\
\hline Diazinón & $\begin{array}{l}\text { Dragon } 25 \% \text {, Basudin } 600, \\
\text { EC } 60 \% \text {, Spectracide }\end{array}$ & & $\begin{array}{l}\text { II / } 300 \mathrm{mg} / \mathrm{kg} \\
\text { (moderadamente peligroso) }\end{array}$ \\
\hline Malatión & Malation, Cythion & 57.8 & \begin{tabular}{|l|} 
III / $2100 \mathrm{mg} / \mathrm{kg}$ \\
(ligeramente peligroso)
\end{tabular} \\
\hline Etión & Etion, Ethanox & & $\begin{array}{l}\text { II / } 208 \mathrm{mg} / \mathrm{kg} \\
\text { (moderadamente peligroso) }\end{array}$ \\
\hline Tebupirimfos & Azteca 2000, Granulado $20 \%$ & & $\begin{array}{l}\text { IA / } 1.3 \mathrm{mg} / \mathrm{kg} \\
\text { (extremadamente peligroso) }\end{array}$ \\
\hline Terbufos & $\begin{array}{l}\text { Triunfo } 5 \% \text {, Vikingo } 5 \% \text {, } \\
\text { Counter } 15 \% \text {, Acance } 5 \% \text {, Counter }\end{array}$ & 76.6 & $\begin{array}{l}\text { IA / } 2 \mathrm{mg} / \mathrm{kg} \\
\text { (extremadamente peligroso) }\end{array}$ \\
\hline
\end{tabular}

( ${ }^{\mathrm{a}}$ Bautista-Ávalos et al., 2014; $\left.{ }^{\mathrm{b}} \mathrm{WHO}, 2009\right)$ 
De los insecticidas OF manejados en la región Ciénega de Chapala se seleccionaron 2 de los más empleados por los agricultores, siendo estos el clorpirifos-etílico y el malatión. En la primera etapa de análisis, se examinaron los factores de retención (RF) y la separación correspondiente del clorpirifos-etílico y del malatión bajo las condiciones establecidas de HPTLC. En la Figura 1, se muestran los resultados del análisis de separación bajo un gradiente de volumen. Las pistas del 1 al 11 corresponden a las disoluciones estándar de clorpirifos-etílico en un gradiente de menor a mayor concentración $(0.25,0.50,0.75,1.00,2.5,5.0,7.5,10,25,50$ y $100 \mu \mathrm{g}$, respectivamente); mientras que los puntos del 12 al 20 corresponden a las disoluciones estándar de malatión $(0.25,0.50,0.75,1.00,2.5,5.0,7.5,10$ y $25 \mu \mathrm{g}$, respectivamente). Se aprecia que los RF del clorpirifos-etílico y malatión para los diferentes volúmenes oscilaron entre 0.73 y 0.63 , respectivamente; además, se observa claramente que la intensidad de las manchas está directamente relacionada con la concentración aplicada. Estos resultados son consistentes con los de otros estudios y sugieren que la cromatografía de capa fina de alta resolución puede ser efectiva para determinar de forma rápida estos 2 compuestos $\mathrm{OF}$ bajo este sistema analítico. El valor del RF es característico de cada compuesto y de utilidad para su identificación, ya que permite compararlo con el RF de su estándar y de otros componentes del extracto separados en la misma placa; asimismo, el color o la fluorescencia de las manchas puede ayudar a su identificación.

Lo visualizado en la Figura 1 se complementa con la información del Cuadro 2 que muestra los valores de los RF obtenidos en este estudio para el clorpirifos-etílico y el malatión, específicamente, en cada una de las pistas aplicadas. Los valores promedio del RF obtenidos en esta indagación para el clorpirifos y malatión fueron 0.747 (CV $0.67 \%$ ) y 0.657 (CV 1.24 \%), respectivamente.

Los valores de RF que se reportan varían dependiendo del tipo de placa, disolvente, sistema de detección y de otros factores (Sherma, 2017). Se han reportado diferentes valores del RF para el malatión y clorpirifos-etílico en sistemas de HPTLC sobre placas de silica gel con diferentes sistemas de disolvente como fase móvil. Ambrus et al. (2005) obtuvieron valores de RF: 0.64, 0.43, 0.72 y 0.17 para el malatión con acetato de etilo; n-hexano:éter dietílico (1:2); éter de petroleo:éter dietílico (1:2); éter de petroleo:éter dietílico (5:1), y éter dietílico, respectivamente como fases móviles, mientras que en el caso del clorpirifos los valores de RF fueron: 0.67, 0.60, 0.99 y 0.87 , respectivamente. Jaiswal et al. (2008a) evaluaron diferentes sistemas de disolventes para el análisis de clorpirifos en muestras de sangre, empleando cloruro de paladio como reactivo de pulverización, obteniéndose los siguientes valores del RF: $0.921,0.895,0.921$ y 0.582 con dietil éter:acetona (8:2), dietil éter:hexano (4:6), ciclohexano:acetona (8:2) y ciclohexano:tetracloruro de carbono (1:9), respectivamente. Por otro lado, los valores del RF del malatión en muestras de sangre y orina, fueron: 0.20 con ciclohexano:cloroformo (60:40), 0.46 con hexano:acetona (80:20) y 0.77 con acetona:Hexano:Tolueno (40:40:20) (Jaiswal et al., 2008b). Iqbal et al. (2009) obtuvieron un RF de 0.05 para el clorpirifos en muestras de vegetales, usando acetato de etilo como fase móvil y vapores de bromo rociados con solución de enzima de sangre de caballo para el revelado. Por último, $\mathrm{Mu}$ nawar y Hameed (2013) obtuvieron un RF de 0.669 para el clorpirifos con metanol:amonio como fase móvil y yoduro de potasio y O-toluidina como reactivos reveladores.

Las diferencias en los valores del RF pueden ser explicadas, en parte, por las condiciones específicas del sistema de solventes y de la fase móvil utilizada; también hay que tomar en cuenta

Creative Commons (Reconocimiento al autor-No comercial-Compartir igual 4.0 Internacional
(CC BY NC SA 4.0 Internacional)


el porcentaje de humedad relativa en la cámara de desarrollo, entre otras variables. Hará falta acometer más estudios que tomen en cuenta estas variables, para definir los valores de RF específicos de estas sustancias.

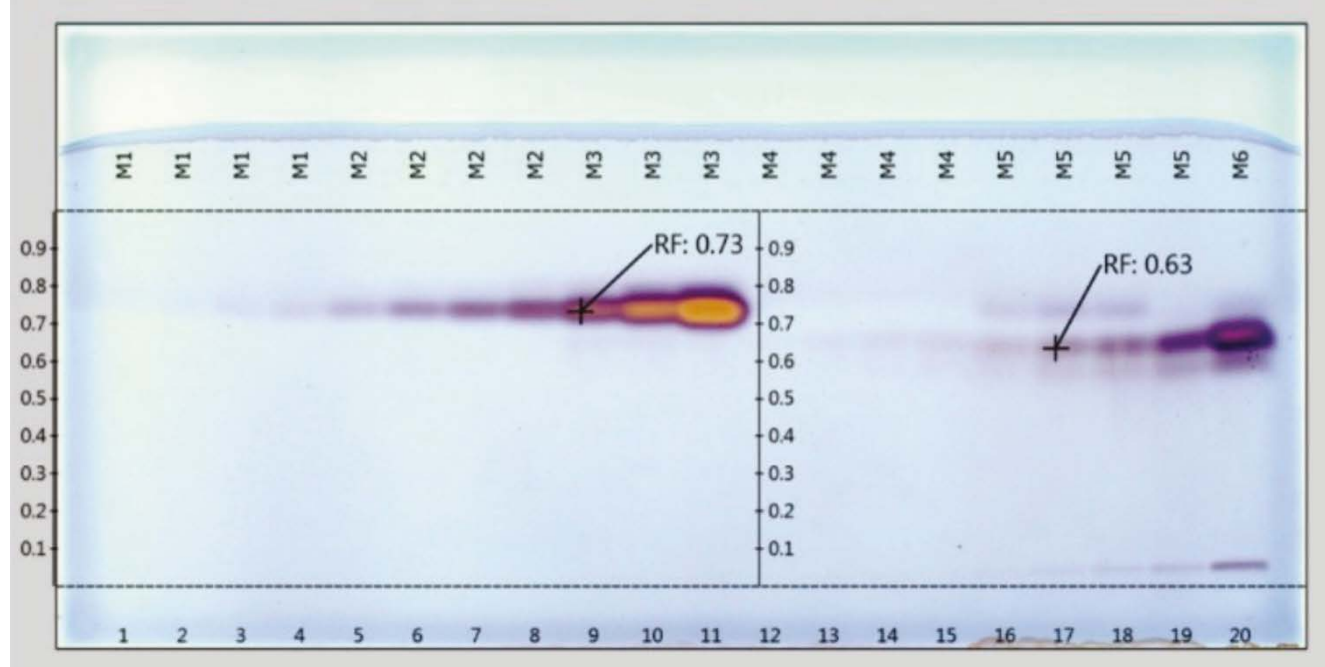

Figura 1. Placa HPTLC de silica gel 60 de clorpirifos-etílico y malatión en disoluciones patrón a diferentes concentraciones, revelada con cloruro de paladio y observada bajo luz blanca

Cuadro2. Valores de RF del clorpirifos-etílico y malatión obtenidos del análisis de disoluciones estándar

\begin{tabular}{c|c|c|c}
\hline \multirow{2}{*}{ Nivel } & $\begin{array}{c}\text { Concentración } \\
\mu g\end{array}$ & Clorpirifos & Malatión \\
\hline & & - & - \\
\hline 1 & 0.25 & - & - \\
\hline 2 & 0.50 & 0.74 & - \\
\hline 3 & 0.75 & 0.74 & 0.65 \\
\hline 4 & 1.00 & 0.74 & 0.66 \\
\hline 5 & 2.5 & 0.75 & 0.65 \\
\hline 6 & 5.0 & 0.75 & 0.65 \\
\hline 7 & 7.5 & 0.75 & 0.66 \\
\hline 8 & 10 & 0.75 & 0.67 \\
\hline 9 & 25 & 0.75 & - \\
\hline 10 & 50 & 0.75 & 0.657 \\
\hline 11 & 100 & 0.747 &
\end{tabular}


La observación individual más impactante que surgió de la comparación de resultados fueron los perfiles de concentración de las disoluciones estándar de clorpirifos-etílico y malatión obtenidos (Figura 2). Se puede ver que los puntos del 1 al 11 corresponden a las disoluciones de clorpirifos-etílico $(0.25,0.50,0.75,1.00,2.5,5.0,7.5,10,25,50$ y $100 \mu \mathrm{g})$, y los puntos del 12 al 20 a las disoluciones de malatión $(0.25,0.50,0.75,1.00,2.5,5.0,7.5,10$ y $25 \mu \mathrm{g})$. La altura y área del pico es proporcional a la concentración del plaguicida.

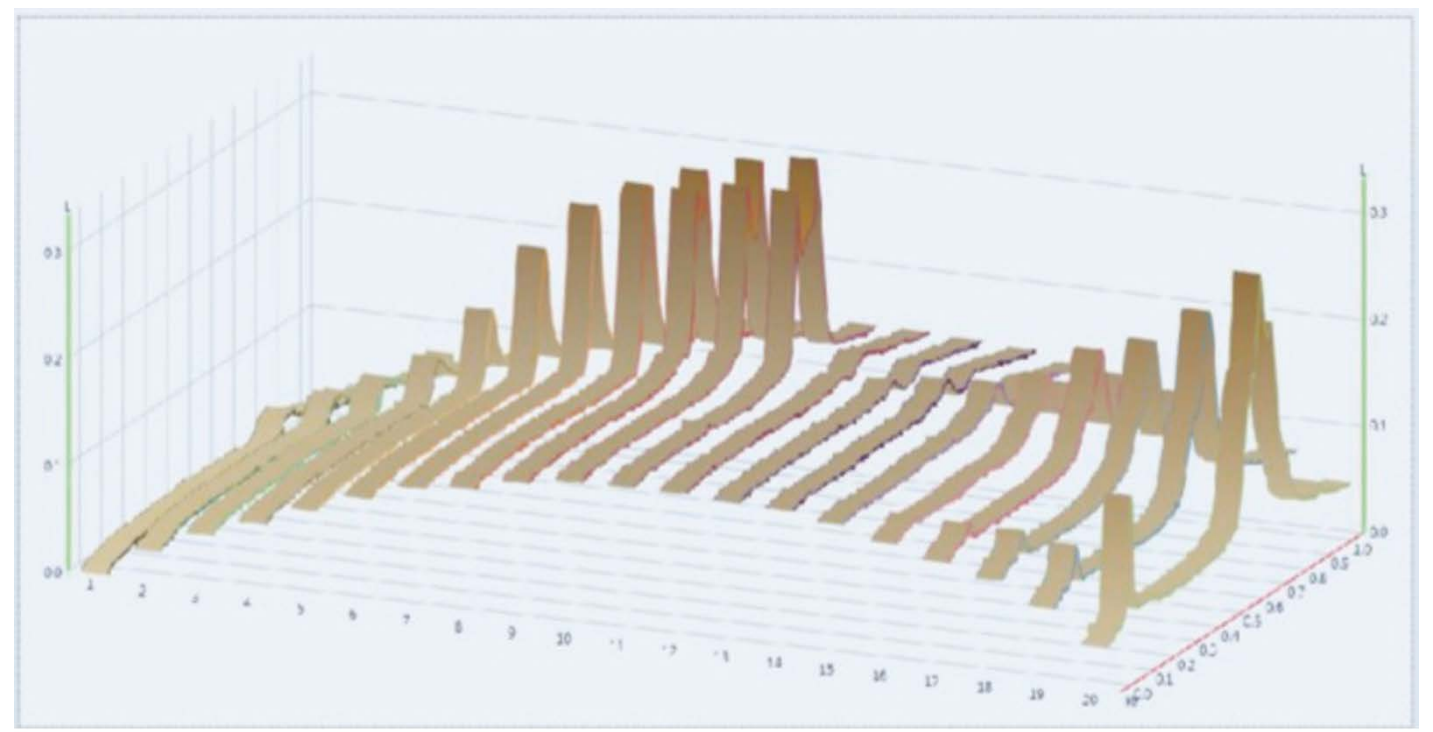

Figura 2. Perfiles de concentración de las disoluciones estándar de clorpirifos-etílico y malatión obtenidos por HPTLC

En las Figuras 3 y 4 se presentan 2 placas que corresponden a los resultados obtenidos de las muestras de vegetales analizadas por HPTLC en este estudio. Se presentan 19 pistas por placa, en las número 1 y 11 , se aplicó una solución estándar de referencia correspondiente al clorpirifos-etílico, mientas que en las 2 y 12, una solución de referencia de malatión, y en el resto de las pistas, se pueden observar las separaciones de los extractos de las muestras recolectadas de material vegetal. Así mismo, se notan manchas de color marrón en forma de una banda, en las pistas de los estándares de referencia con un RF de 0.747 y 0.657 para el clorpirifos y malatión, respectivamente.

\begin{tabular}{|c|c|c|}
\hline 48 & $\begin{array}{l}\text { Creative Commons (Reconocimiento al autor-No comercial-Compartir igual } 4.0 \text { Internacional } \\
\text { (CC BY NC SA } 4.0 \text { Internacional) }\end{array}$ & $\begin{array}{l}\text { Revista de } \\
\text { CIENCIAS AMBIENTALES } \\
\text { Tropical Journal of Environmental Sciences }\end{array}$ \\
\hline
\end{tabular}




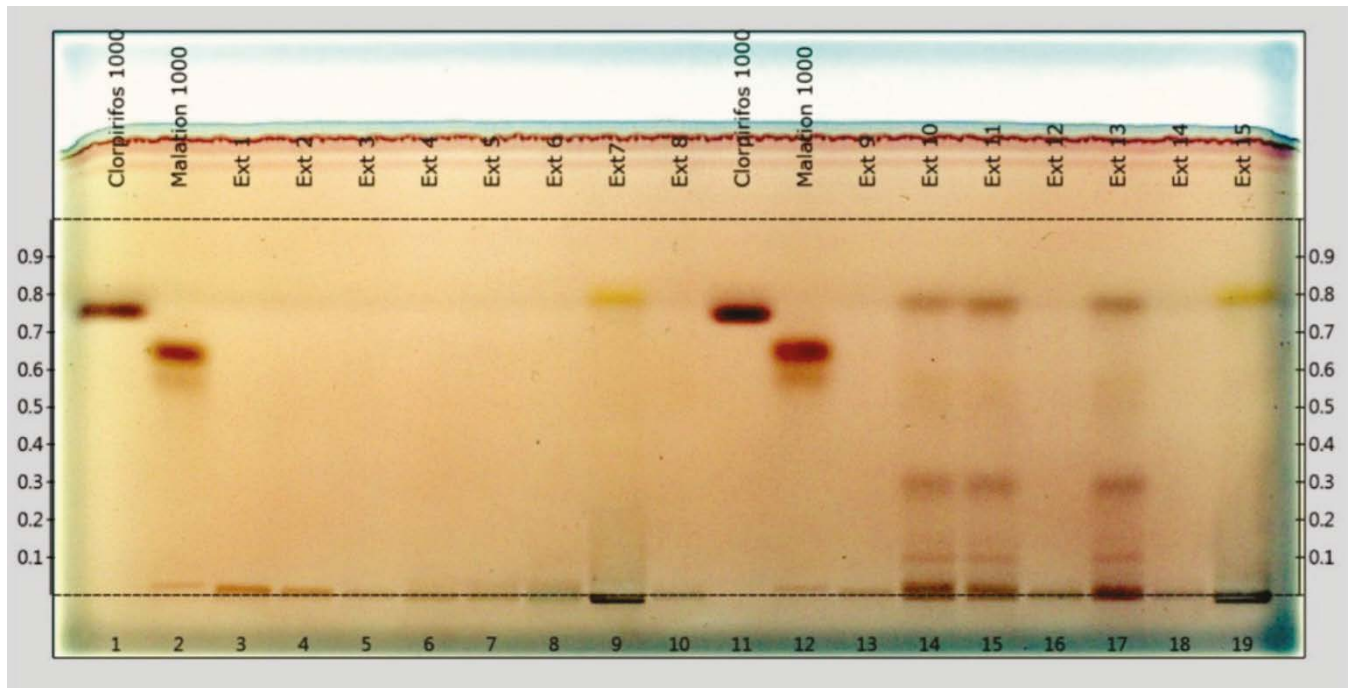

Figura 3. Extractos de muestras de vegetales (placa 1) analizadas por HPTLC en un sistema de gel de sílice 60-hexano:acetona (8:2), revelada con cloruro de paladio y observada bajo luz blanca

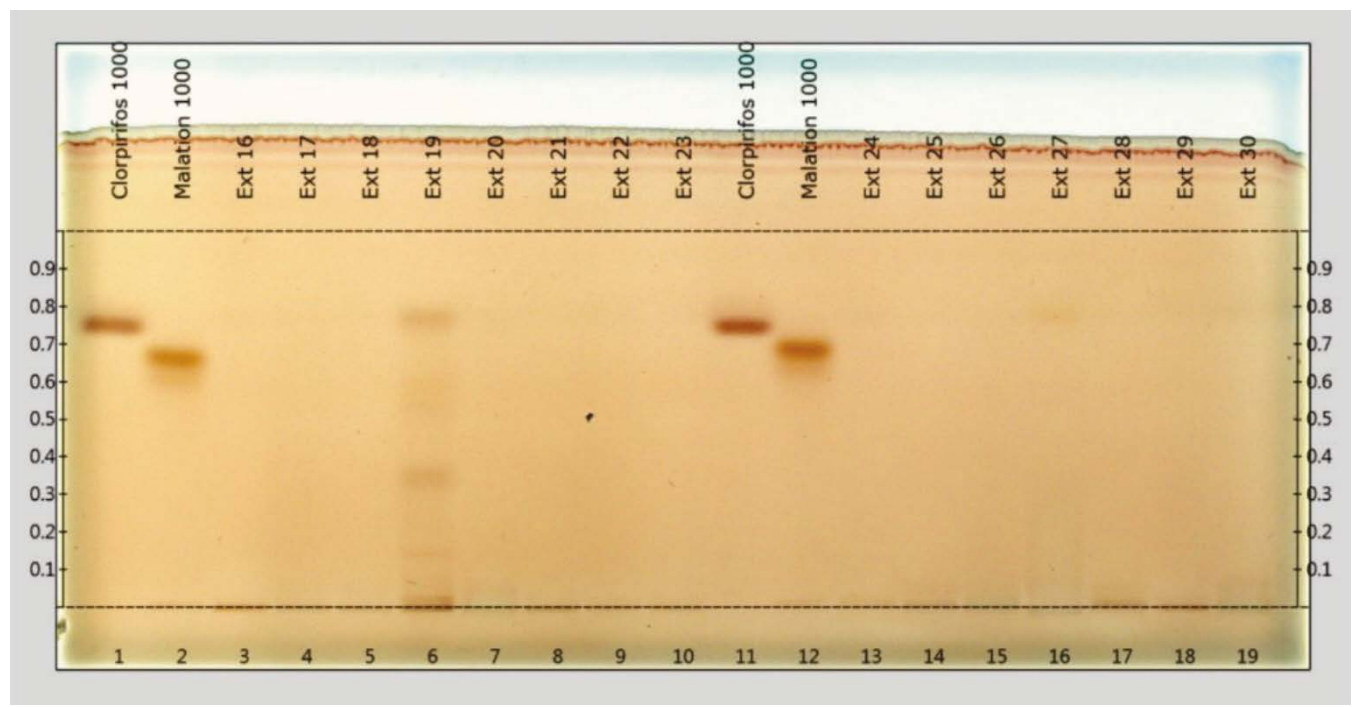

Figura 4. Extractos de muestras de vegetales (placa 2) analizadas por HPTLC en un sistema de gel de sílice 60-hexano:acetona (8:2), revelada con cloruro de paladio y observada bajo luz blanca 
En todas las muestras analizadas, no se detectaron residuos de malatión. Sin embargo, se observó la presencia de residuos de clorpirifos-etílico en el $20 \%$ de las muestras, en 4 muestras de cebolla y en 1 de manzana (Cuadro 3). Las de cebolla que dieron positivo fueron 2 del tianguis ubicado a un costado del Jardín de la Paz, 1 del tianguis que se establece en el centro de la localidad y 1 del tianguis situado cerca del Estadio. La muestra de manzana procedía del tianguis en el centro de la localidad.

Respecto al malatión, no se detectaron residuos de plaguicidas en ningunas de las muestras de vegetales recolectadas en los tianguis y en el mercado local. Por otro lado, en las muestras orgánicas de jitomate y fresa, no se detectó la presencia de residuos de clorpirifos-etílico ni malatión. Al comparar la intensidad de las bandas de las muestras de vegetales que resultaron positivas para el clorpirifos-etílico respecto a las bandas de las disoluciones estándar, se estima que las concentraciones detectadas en las muestras podrían estar por debajo de $1 \mathrm{mg} / \mathrm{kg}$.

El procedimiento de lavado realizado no eliminó los residuos de plaguicidas, ya que se detectó la presencia de ellos en algunos de los vegetales. Se ha reportado que los plaguicidas que tienen una solubilidad baja en agua podrían permanecer en la superficie de los alimentos, aún después del lavado (Gil et al., 2009). En el caso del clorpirifos, su solubilidad en agua es baja (1.4 mg/L a 25 ${ }^{\circ} \mathrm{C}$ ). En México, pocos estudios han determinado residuos de plaguicidas $\mathrm{OF}$ en vegetales. 
Cuadro 3. Residuos de clorpirifos-etílico en las muestras de vegetales analizadas

\begin{tabular}{|c|c|c|c|c|c|}
\hline No. en la placa & Placa & No. de extracto & Tianguis & RF & Presencia de clorpirifos \\
\hline 3 & 1 & 1 & $\mathrm{TC}$ & & - \\
\hline 4 & 1 & 2 & $\mathrm{TJ}$ & & - \\
\hline 5 & 1 & 3 & TC & & - \\
\hline 6 & 1 & 4 & $\mathrm{TC}$ & & - \\
\hline 7 & 1 & 5 & $\mathrm{TJ}$ & & - \\
\hline 8 & 1 & 6 & TC & & - \\
\hline 9 & 1 & 7 & TC & 0.78 & $+/-$ \\
\hline 10 & 1 & 8 & $\mathrm{M}$ & & - \\
\hline 13 & 1 & 9 & -- & & - \\
\hline 14 & 1 & 10 & $\mathrm{TC}$ & 0.77 & + \\
\hline 15 & 1 & 11 & $\mathrm{TJ}$ & 0.77 & + \\
\hline 16 & 1 & 12 & $\mathrm{TJ}$ & & - \\
\hline 17 & 1 & 13 & $\mathrm{TE}$ & 0.77 & + \\
\hline 18 & 1 & 14 & -- & & - \\
\hline 19 & 1 & 15 & $\mathrm{TJ}$ & 0.78 & $+/-$ \\
\hline 3 & 2 & 16 & $\mathrm{TE}$ & & - \\
\hline 4 & 2 & 17 & -- & & - \\
\hline 5 & 2 & 18 & -- & & - \\
\hline 6 & 2 & 19 & $\mathrm{M}$ & & - \\
\hline 7 & 2 & 20 & $\mathrm{TJ}$ & & - \\
\hline 8 & 2 & 21 & $\mathrm{TE}$ & & - \\
\hline 9 & 2 & 22 & -- & & - \\
\hline 10 & 2 & 23 & $\mathrm{M}$ & & - \\
\hline 13 & 2 & 24 & -- & & - \\
\hline 14 & 2 & 25 & $\mathrm{TE}$ & & - \\
\hline 15 & 2 & 26 & $\mathrm{TE}$ & & - \\
\hline 16 & 2 & 27 & $\mathrm{TE}$ & & $+/--$ \\
\hline 17 & 2 & 28 & $\mathrm{M}$ & & - \\
\hline 18 & 2 & 29 & $\mathrm{M}$ & & - \\
\hline 19 & 2 & 30 & $\mathrm{M}$ & & - \\
\hline
\end{tabular}


Revista de Ciencias Ambientales (Trop J Environ Sci). (Julio-Diciembre, 2018). EISSN: 2215-3896. Vol 52(2): 39-56.

DOI: http://dx.doi.org/10.15359/rca.52-2.3

URL: www.revistas.una.ac.cr/ambientales

EMAlL: revista.ambientales@una.cr

El CNRPYC ha realizado monitoreos anuales de residuos de plaguicidas en cultivos agrícolas comerciales en varios estados de México. Los resultados de estos monitoreos evidencian que se siguen aplicando OF prohibidos en otros países (Cuadro 4). En el 2005, se encontraron 248 muestras con la presencia de al menos 1 residuo, 33 muestras con 2 y 25 con 3 . Alrededor del 30 $\%$ de las muestras no presentó residuos de plaguicidas.

Cuadro 4. Insecticidas organofosforados no autorizados o restringidos detectados en los monitoreos 2005-2007 del CNRPYC

\begin{tabular}{|c|c|c|c|}
\hline \multirow{2}{*}{ OF } & \multicolumn{3}{|c|}{ Cultivos en los que se detectó } \\
\hline & 2005 & 2006 & 2007 \\
\hline Acefato & Tomate y papaya & Jitomate y cilantro & Jitomate y calabacita \\
\hline Clorpirifos-etílico & Chile, apio, lechuga, nopal y cilantro & Brocoli, lechuga y guayaba & Cilantro, brócoli, col y guayaba \\
\hline Diazinón & Cilantro & & \\
\hline Diclorvos & Fresa & & \\
\hline Dicrotofos & Apio & & \\
\hline Dimeotato & Nopal y cilantro & Guayaba & Papaya, cilantro y calabacita \\
\hline Etión & & & Guayaba \\
\hline Metamidofos & Fresa y frijol ejotero & Fresa, cilantro y guayaba & Cilantro y papaya \\
\hline Monocrotofos & Chile y tomate & Chile y tomate & Chile y cilantro \\
\hline Ometoato & Chile, apio, frijol, lechuga y nopal & Chile, lechuga, tomate y guayaba & $\begin{array}{l}\text { Chile, lechuga, tomate, papaya, } \\
\text { cilantro y calabacita }\end{array}$ \\
\hline Paratión metílico & Chile, nopal y frijos elotero & Chile, lechuga, tomate y guayaba & $\begin{array}{l}\text { Chile, lechuga, tomate, papaya, } \\
\text { cilantro y calabacita }\end{array}$ \\
\hline Profenofos & & & Tomate \\
\hline
\end{tabular}

Elaborado a partir de Pérez et al. (2013)

Hernández-Hernández et al. (2007) encontraron residuos de clorpirifos y malatión en cultivos de papaya en concentraciones de $0.8 \pm 0.5$ y $2.4 \pm 1.9 \mu \mathrm{g} / \mathrm{kg}$, respectivamente, y Pérez et al. (2009) reportaron residuos de clorfenvinfos, malatión, diazinón, fentión y etión en brócoli, en concentraciones de 5.78, 2.67, 1.16, 0.041 y $0.024 \mathrm{mg} / \mathrm{kg}$, respectivamente. Diversas organizaciones internacionales y gobiernos de países llevan a cabo programas periódicos de monitoreo de residuos de plaguicidas para vigilar la calidad e inocuidad de los alimentos y garantizar que no se rebasen los LMR (Hjorth et al., 2011; Skretteberg et al., 2015). En México, los LMRs son menos estrictos que los de la UE, el Codex Alimentarius y Japón (Cuadro 5). 
Revista de Ciencias Ambientales (Trop J Environ Sci). (Julio-Diciembre, 2018). EISSN: 2215-3896. Vol 52(2): 39-56.

DOI: http://dx.doi.org/10.15359/rca.52-2.3

URL: www.revistas.una.ac.cr/ambientales EMAIL: revista.ambientales@una.cr

Cuadro 5. LMR (mg/kg) de residuos de plaguicidas organofosforados

\begin{tabular}{|c|c|c|c|c|c|}
\hline OF & México 2007 & EUA & UE & Codex Alimentarius & Japón \\
\hline Paratión metílico & $0.1-1.0$ & $0.2-1$ & 0.01 & $0.05-0.3$ & $0.1-1$ \\
\hline Clorpirifos & $0.5-1$ & $0.05-2$ & $0.05-1$ & $0.01-2$ & $0.01-1$ \\
\hline Clorpirifos etílico & $0.05-3$ & 6 & - & - & - \\
\hline Metamidofos & $0.05-2$ & $0.1-1$ & 0.01 & $0.02-0.050$ & $0.01-1$ \\
\hline Diazinón & $\begin{array}{c}0.1-0.75 \\
0.1-40\end{array}$ & $\begin{array}{c}0.1-0.75 \\
0.1-1.0\end{array}$ & $0.01-0.05$ & $\begin{array}{l}0.05-0.5 \\
0.01-5.0\end{array}$ & $\begin{array}{l}0.05-0.1 \\
0.02-0.7\end{array}$ \\
\hline Omeotato & $0.05-2$ & - & - & $0.01-0.05$ & $0.7-2$ \\
\hline Etión & $0.1-2.0$ & $2-10$ & 0.01 & $0.3-5$ & $0.1-1$ \\
\hline Dicrotofos & 0.2 & 0.05 & - & 0.05 & 0.05 \\
\hline Diclorvos & 0.5 & 0.5 & 0.01 & - & 0.1 \\
\hline Monocrotofos & $0.02-0.1$ & - & 0.01 & - & $0.05-0.2$ \\
\hline Acefato & $0.5-10$ & $4-10$ & 0.01 & $1-50$ & $0.1-5$ \\
\hline Dimetoato & $0.05-2.0$ & $0.2-2$ & $0.02-0.1$ & $0.05-1$ & $0.2-1$ \\
\hline
\end{tabular}

Elaborado a partir de Pérez et al. (2013)

La cebolla es una de las hortalizas de mayor importancia en el consumo humano. En el nivel nacional, Michoacán ocupa el séptimo lugar en superficie sembrada de cebolla, destacándose los municipios de Villamar, Yurécuaro y Jacona. En algunos de estos últimos, se ha reportado el uso de plaguicidas no recomendados como el clorpirifos y el metamidofos para el control fitosanitario de plagas en el cultivo de cebolla. Respecto al cultivo de manzana, Michoacán ocupa el décimo lugar en superficie sembrada, y los principales municipios que la producen son: Zitácuaro, Morelia, Uruapan, Pátzcuaro y Sahuayo. En México, el uso del malatión está autorizado para los cultivos de cebolla y manzana, y el LMR es de $8 \mathrm{mg} / \mathrm{kg}$; en el caso del clorpirifos-etílico, está autorizado para el cultivo de manzana con un LMR de $1.5 \mathrm{mg} / \mathrm{kg}$ (COFEPRIS, 2016).

El empleo de plaguicidas en cultivos agrícolas debe realizarse conforme a LMR de normas de inocuidad, con el fin de asegurar que los riesgos para los consumidores sean mínimos. Sin embargo, se debe considerar que, aunque no se rebasen los LMR de ciertos plaguicidas en los alimentos, podría existir un efecto aditivo por la exposición crónica a dichos compuestos, presentándose posibles efectos adversos a la salud humana, a largo plazo.

Actualmente, en la región de estudio se utilizan varios OF para el control de plagas en frutas y verduras; sin embargo, este estudio se limitó solo a la detección de clorpirifos-etílico y malatión, en un un nivel exploratorio, en ciertos tipos de frutas y vegetales de los tianguis y del mercado de la localidad. Por lo anterior, se requiere realizar un estudio estacional que incluya un mayor número y tipo de plaguicidas, un grupo más amplio de variedades de frutas y vegetales, una cantidad de muestras más grande, la aplicación de encuestas para conocer con precisión la procedencia de las muestras (local, nacional o de importación) de frutas y vegetales, así como las cuantías de plaguicidas que se aplican a los cultivos. También es importante el empleo de otras técnicas analíticas confirmatorias. 


\section{Conclusiones}

A pesar de las limitaciones de este estudio, los resultados obtenidos indican la presencia de residuos de OF en frutas y vegetales que se comercializan en la localidad. Se estima que las concentraciones de clorpirifos-etílico podrían estar por debajo de los LMR; sin embargo, se debe considerar que, además del clorpirifos, la población puede estar expuesta a más OF y a plaguicidas de otros grupos químicos, lo que representa un riesgo, debido a que los efectos dañinos pueden ser aditivos y manifestarse a largo plazo. En el caso del clorpirifos, este OF afecta los sistemas nervioso central, cardiovascular y respiratorio. Dada la toxicidad y peligrosidad de muchos plaguicidas que se emplean de manera intensiva en el control de plagas en los campos agrícolas mexicanos, se requiere el monitoreo periódico de residuos de plaguicidas en alimentos, matrices ambientales y la población en general ocupacionalmente expuesta.

\section{Agradecimientos}

El presente trabajo fue realizado con financiamiento y apoyo del Instituto Politécnico Nacional a través del proyecto Determinación de plaguicidas en vegetales producidos y consumidos en la región Ciénega de Chapala. Estudio preliminar. Clave SIP: 20162199. Agradecemos a la revista y las personas revisoras anónimas por las observaciones realizadas al documento.

\section{Referencias}

Ambrus, A., Füzesi, I., Susán, M., Dobi, D., Lantos, J., Zakar, F., Korsós, I., Oláh, J., Beke, B. B. y Katavics, L. (2005). A cost-effective screening method for pesticide residue analysis in fruits, vegetables, and cereal grains. J. Environ. Sci. Health. B., 40(2), 297-339.

Androutsopoulos, V. P., Hernández, A. F., Liesivuori, J. y Tsatsakis, A. M. (2012). A mechanistic overview of health associated effects of low levels of organochlorine and organophosphorous pesticides. Toxicology, 307, 89-94.

Bautista-Ávalos, D., Cruz-Cárdenas, G., Moncayo-Estrada, R., Silva-García, J. T. y Estrada-Godoy, F. (2014). Aplicación del modelo SWAT para evaluar la contaminación por fuentes difusas en la subcuenca del lago de Chapala, México. Rev. Int. Contam. Ambie., 30(3), 263-274.

Bouchard, M. F., Chevrier, J., Harley, K. G., Kogut, K., Vedar, M., Calderon, N. et al. (2011). Prenatal Exposure to Organophosphate Pesticides and IQ in 7-Year-Old Children. Environ. Health Perspect., 119, 1189-1195.

Chen, C., Qian, Y. Z., Liu, X. J., Tao, C. J., Liang, Y. y Li, Y. (2012). Risk assessment of chlorpyrifos on rice and cabbage in China. Regul. Toxicol. Pharm., 62, 125-130.

Comisión Federal para la Protección contra Riesgos Sanitarios [COFEPRIS]. (2016). Catálogo de Plaguicidas. México. Recuperado de http://www.cofepris.gob.mx/AZ/Paginas/Plaguicidas\%20y\%20Fertilizantes/CatalogoPlaguicidas.aspx 
Damalas, C. A. y Eleftherohorinos, I.G. (2011). Pesticide Exposure, Safety Issues, and Risk Assessment Indicators. Int. J. Environ. Res. Public Health, 8, 1402-1419.

Duhau, E. y Giglia, A. (2007). Nuevas centralidades y prácticas de consumo en la Ciudad de México: del microcomercio al hipermercado. EURE (Santiago), 33(98), 77-95.

EFSA [European Food Safety Authority]. (2017). The 2015 European Union Report on Pesticide. Residues in Food. EFSA Journal, 15(4), 4791, 1-134.

Fenik, J., Tankiewicz, M. y Biziuk, M. (2011). Properties and determination of pesticides in fruits and vegetables. Trends in Analytical Chemistry, 30(6), 814-826.

Food and Drug Administration [FDA]. (2015). Pesticide Residue Monitoring Program, Fiscal Year 2015 Pesticide Report. USA, 1-47.

Gil, M. I., Selma, M. V., López-Gálvez, F. y Allende, A. (2009). Fresh-cut product sanitation and ash water disinfection: Problems and solutions. International Journal of Food Microbiology, $134,37-45$.

Hernández-Antonio, A. y Hansen, A. M. (2011). Uso de plaguicidas en dos zonas agrícolas de México y evaluación de la contaminación de agua y sedimentos. Revista internacional de contaminación ambiental, 27(2), 115-127.

Hernández-Hernández, C. N. A., Valle-Mora, J., Santiesteban-Hernández, A. y Bello-Mendoza, R. (2007). Comparative ecological risks of pesticides used in plantation production of papaya: Application of the SYNOPS indicator. Science of the Total Environment, 381(1-3), 112-125.

Hjorth, K., Johansen, K., Holen, B., Andersson, A., Christensen, H. B., Siivinen, K. y Toome, M. (2011). Pesticide residues in fruits and vegetables from South America - A Nordic project. Food Control, 22, 1701-1706.

Instituto Nacional de Estadística y Geografía [INEGI]. (2017). Anuario estadístico y geográfico de Michoacán de Ocampo 2017. Recuperado de http://www.beta.inegi.org.mx/app/buscador/default.html?q=anuario+estadistico+de+michoacan

Iqbal, M. F., Maqbool, U., Perveez, I., Farooq, M. y Asi, M. R. (2009). Monitoring of insecticide residues in Brinjal collected from market of Noshera Virkan, Pakistan. The Journal of Animal \& Plant Sciences, 19(2), 90-93.

Jaiswal, A. K., Giri, N. G., Gupta, M., Kumar, P. y Rejectpaul, M. (2008a). Development of new solvent systems for the analysis of chlorpirifos poison extracted from blood. Proc. Indian Natn. Sci. Acad., 74(4), 173-177.

Jaiswal, A. K., Kaur, A., Millo, T., Kumar, A. y Gupta, M. (2008b). Determination Of Malathion In Blood And Urine Using Thin-Layer Chromatography. J. Indian Acad. Forensic Med., 30(1), 15-21. 
Kazemi, M., Tahmasbi, A. M., Valizadeh, R., Naserian, A. A. y Soni, A. (2012). Organophosphate pesticides: a general review. Agric. Sci. Res. J., 2(9), 512-529.

Mostafalou, S. y Abdollahi, M. (2013). Pesticides and human chronic diseases: evidences, mechanisms, and perspectives. Toxicol Appl Pharmacol., 268(2),157-177.

Munawar, A. y Hameed, S. W. (2013). Quantification of Pesticide Residues in Vegetables by Different Chromatographic Techniques. J. Chromatograph. Separat. Techniq., 4-8. Recuperado de http://dx.doi.org/10.4172/2157-7064.1000200

Obare, S. O., De, C., Guo, W., Haywood, T. L., Samuels, T. A., Adams, C. P., Masika, N.O., Murray, D. H., Anderson, G. A., Campbell, K. y Fletcher, K. (2010). Fluorescent Chemosensors for Toxic Organophosphorus Pesticides: A Review. Sensors, 10, 7018-7043.

Pérez, M. A., Navarro, H. y Miranda, E. (2013). Residuos de plaguicidas en hortalizas: problemática y riesgo en México. Rev. Int. Contam. Ambie., 29 (número especial sobre plaguicidas), 45-64.

Pérez, M. A., Segura, A., García, R., Colinas, T., Pérez, M., Vázquez, A. y Navarro, H. (2009). Residuos de plaguicidas organofosforados en cabezuela de brócoli (brassica oleracea) determinados por cromatografía de gases. Rev. Int. Contam. Ambie., 25(2), 103-109.

Pérez-Olvera, M. A., Navarro-Garza, H. y Miranda-Cruz, E. (2011). Use of pesticides for vegetable crops in Mexico. En M. Stoytcheva (ed.), Pesticides in the Modern World-Pesticides Use and Management. InTech. Rijeka, Croatia., 97-118.

Sandoval-Moreno, A. y Ochoa, M. A. (2010). Grupos locales, acceso al agua y su problemática de contaminación en la Ciénega de Chapala, Mich., Economía, sociedad y territorio., 10(34), 683-719.

Sherma, J. (2017). Review of thin-layer chromatography in pesticide analysis: 2014-2016. Journal of Liquid Chromatography \& Related Technologies, 40(5-6), 226-238

Skretteberg, L. G., Lyrån, B., Holen, B., Jansson, A., Fohgelberg, P., Siivinen, K., Andersen, J. H. y Jensen, B. H. (2015). Pesticide residues in food of plant origin from Southeast Asia e A Nordic project. Food Control, 51, 225-235.

Suárez-Jacobo, A., Alcantar-Rosales, V. M., Alonso-Segura, D., Heras-Ramírez, M., Elizarragaz-De La Rosa, D., Lugo-Melchor, O. y Gaspar-Ramírez, O. (2017). Pesticide residues in orange fruit from citrus orchards in Nuevo Leon State, Mexico. Food Additives \& Contaminants: Part B, 10(3), 192-199.

World Health Organization (WHO). (2009). The WHO recommended classification of pesticides by hazard and guidelines to classification. WHO Publications, Geneva, Switzerland. 